CARDIOVASCULAR MEDICINE

\title{
Long term follow up of children with myocarditis treated by immunosuppression and of children with dilated cardiomyopathy
}

\author{
M Giulia Gagliardi, M Bevilacqua, C Bassano, B Leonardi, R Boldrini, F Diomedi Camassei, \\ A Fierabracci, A G Ugazio, G F Bottazzo
}

Heart 2004;90:1167-1171. doi: 10.1136/hrt.2003.026641

\begin{abstract}
See end of article for authors' affiliations

Correspondence to Dr Maria G Gagliardi, Dipartimento MedicoChirurgico di Cardiologia, Ospedale Pediatrico Bambino Gesù, IRCCS Piazza S Onofrio 4, 00165 Rome, Italy; gagliard@opbg.net
\end{abstract}

Accepted 25 March 2004

\begin{abstract}
Objective: To describe the treatment and long term outcome after immunosuppressive treatment of children with myocarditis.

Methods and results: 114 patients with newly diagnosed dilated cardiomyopathy were divided into three groups, according to the histological pattern: group A, acute myocarditis; group B, borderline myocarditis; and group C, non-inflammatory cardiomyopathy. Groups A and B were treated with cyclosporine and prednisone in addition to conventional treatment. Survivors of the whole cohort were analysed for 13 year transplant-free survival and assessed for left ventricular function. Event-free survival at 13 years was $97(3) \%$ for group A, 70 (8)\% for group B, and $32(7) \%$ for group C ( $<<0.0001)$. It was $96(4) \%$ at one year and $83(5) \%$ at 13 years for the cumulated myocarditis group (A and B). Cardiac function recovered completely in $79 \%$ of survivors in group A, $64 \%$ in group B, and $36 \%$ in group $C$. The rate of complete recovery in the cumulated group ( $A$ and $B$ ) was $70 \%$.

Conclusions: The high long term survival rate of this cohort of children with myocarditis is probably due to the effect of short term immunosuppression. This result differs from previously published series of conventionally treated children, whose survival probability at one year was about $60 \%$.
\end{abstract}

$\mathrm{D}$ lated cardiomyopathy (DCM) is characterised by an enlarged left ventricle with reduced contractility. The pathogenesis of DCM remains unknown, even though viral infections and autoimmunity have been repeatedly implicated as possible causes of damage to the cardiac myocytes. ${ }^{12}$

After the introduction of endomyocardial biopsy (EMB) into clinical practice in the mid 1980s, a histological classification was proposed (Dallas criteria) ${ }^{3}$ identifying three forms of DCM: acute florid myocarditis, borderline myocarditis, and non-inflammatory cardiomyopathy. The first two forms are characterised by the presence of a mononuclear cell infiltration and the third only by fibrosis with no apparent signs of inflammatory infiltration.

In children with DCM, conventional treatment yielded an overall one year survival of $40-75 \%$ and a five year survival of $37-47 \%{ }^{4-6}$ Those with biopsy proven myocarditis tend to fare better than those with fibrosis seen on biopsy. Among adult patients with DCM, also treated conventionally, there were no survival differences between those with and those without biopsy proven myocarditis, with an event-free survival rate of about $50 \%$ at five years for both groups. ${ }^{7}$

Animal data suggest a role for autoimmunity alone or secondary to viral infection in the damage to cardiac myocytes. ${ }^{8-11}$ Consequently, some centres have reported use of immunosuppression to treat myocarditis. In the first study, ${ }^{12}$ adult patients with DCM presenting with (reactive) or without cardiac inflammation (non-reactive) were randomly assigned to steroid or to conventional treatment. In the second study, ${ }^{13}$ only adults with myocarditis were randomly assigned to immunosuppression or conventional treatment. Although no differences were observed between the two studies in terms of survival, improvement of left ventricular function was reported in the first study ${ }^{14}$ but only in the reactive immunosuppressed group. A single randomised trial has been carried out in children with myocarditis comparing conventional treatment with three different immunosuppressive treatment protocols. ${ }^{15}$ In that study, the association of prednisone with either azathioprine or cyclosporine yielded improved functional recovery and faster histological regression of the inflammatory pattern. Moreover, uncontrolled studies suggest a role for these treatments. Our group ${ }^{15}$ and Kleinert and colleagues ${ }^{16}$ achieved $100 \%$ event-free survival at one year, and Lee and colleagues $^{17}$ reported $86 \%$ event-free survival at two years. Immunomodulation by means of IgG administration was also tried in the treatment of children with myocarditis. ${ }^{18}$ The probability of survival at one year for 21 children was $84 \%$ compared with $60 \%$ for 25 historical controls, who also had myocarditis but were treated only conventionally $(p=0.069)$. In a subsequent study, ${ }^{19}$ adults with DCM who were found to have an upregulation of HLA molecules on $\mathrm{EMB}^{20}$ were randomly assigned to immunosuppression. Although there was no difference in the survival rate between short term immunosuppressed and conventionally treated patients, a long term benefit on left ventricular function was documented in the surviving patients who were immunosuppressed.

We present a 13 year follow up study of 114 children who initially presented with heart failure secondary to DCM. EMB was obtained at diagnosis from all children. Patients with acute florid or borderline myocarditis were assigned to immunosuppression and those with idiopathic DCM were treated conventionally. We present data on the natural history of non-inflammatory DCM among children treated conventionally and the outcome for children with myocarditis

Abbreviations: CHF, congestive heart failure; DCM, dilated cardiomyopathy; EMB, endomyocardial biopsy; LVEDV, left ventricular end diastolic volume indexed to body surface area; LVEF, left ventricular ejection fraction 
treated with immunosuppression. End points of the study were long term event-free survival and the improvement of left ventricular function.

\section{METHODS Study population}

From March 1986 to December 2001, 114 consecutive children with congestive heart failure (CHF) were referred to our department. All had an anatomically normal heart, none had supraventricular arrhythmia, and metabolic disorders that are potential causes of DCM were excluded in all 114 children by conventional laboratory tests carried out in the first three days of admission before they underwent EMB. Table 1 presents their clinical characteristics. Children with less severe symptoms were admitted to the cardiology ward and treated conventionally and patients with severe heart failure went to the intensive care unit and received intravenous treatment. None of our patients had clinical characteristics of fulminant myocarditis. After routine evaluation (taking a personal and family history, physical examination, ECG, and chest radiography), all children underwent an echocardiographic study.

\section{Echocardiographic study}

Left ventricular end diastolic volume indexed to body surface area (LVEDV) and left ventricular ejection fraction (LVEF) were calculated by two dimensional echocardiography, according to the 5/6 area-length formula. ${ }^{21}$ Echocardiography was repeated several times during the acute phase of the disease and then once a month for the following six months. When stabilised, patients had undergone echocardiography twice a year. Less stable patients had a more intense programme with follow up.

To evaluate other parameters predictive of prognosis, we measured the wedge pressure in all patients with a Swan Ganz catheter before the biopsy was taken.

\section{Endomyocardial biopsy}

Once heart failure was controlled (time lapse from admission never exceeding seven days), all patients underwent right cardiac characterisation and EMB. The EMBs were obtained with a 6 French bioptome by the femoral venous or transjugular approach. Four or five tissue samples were obtained from the mid apical portion of the right ventricular septum. The specimens were fixed in a $10 \%$ buffered formalin solution and stained with haematoxylin and eosin for light microscopic evaluation. On the basis of the histological pattern detected, the children were subdivided (by the Dallas criteria) into three groups: group A, those with acute florid myocarditis, group B, those with borderline myocarditis, and group C, those with no myocarditis. Giant cells were not seen in any of the EMBs. In the children from groups $\mathrm{A}$ and $\mathrm{B}$, the biopsy was repeated after six months to monitor the evolution of the histological pattern. Third and fourth EMBs were obtained from patients who continued to have persistent or resolving myocarditis. All biopsy specimens were reviewed blindly by an independent pathologist.

\section{Management and treatment}

All patients had standard circulatory support and underwent a treatment protocol of digoxin, furosemide, and captopril (angiotensin converting enzyme inhibitors were given to a large majority of the patients, starting in 1990). Anticoagulation with warfarin (target international normalised ratio of 2.0-2.5) was started in the entire patient population, regardless of age at onset of DCM, and was withdrawn as soon as left ventricular function improved. On admission, two patients who were subsequently included in group C presented with left ventricular thrombus before anticoagulant treatment was commenced. For children in groups A and B, immunosuppressive treatment was started according to the following protocol: cyclosporine $6-8 \mathrm{mg} / \mathrm{kg} /$ day in two doses, with dose adjustment until the therapeutic blood concentration was reached $\left(170-210 \mathrm{ng} / \mathrm{cm}^{3}\right)$, and prednisone $2 \mathrm{mg} / \mathrm{kg} /$ day in two doses for the first month, tapered thereafter to $0.5 \mathrm{mg} / \mathrm{kg} / \mathrm{day}$ for six months. At that time, a second EMB was obtained. The immunosuppression was discontinued in cases of resolved myocarditis but it was continued for a further six months in the children with persistent or resolving myocarditis. In these patients, a third EMB was obtained at 12 months and immunosuppression discontinued if cardiac function had recovered, regardless of the histological pattern detected on the EMB. Nonetheless, a fourth EMB was obtained at 18 months after the onset of symptoms from most children with a biopsy positive for myocarditis at one year. Children with a sudden return of CHF after suspension of immunosuppression were considered to have recurrent myocarditis. A further EMB was taken and immunosuppression was promptly restarted, regardless of the histological pattern observed. Before the start of the immunosuppression, the parents were informed of possible risks and benefits. The study was approved by the hospital's ethics committee and parental signed consent was obtained for all patients.

\section{Characteristics of the follow up}

The cumulative follow up was 685.3 patient-years and was $98.8 \%$ complete. The mean duration was 6.0 (4.9) years (median 5.7 years, range one month to 15.6 years). Fifty six patients $(46 \%)$ completed six years of follow up, 36 (32\%) 10 years, and six $(5 \%)$ the whole 13 years of follow up. At the follow up visits patients underwent a complete physical examination and an echocardiographic study, aimed at assessing left ventricular function. A reduction of LVEF or

Table 1 Patients' characteristics at first admission

\begin{tabular}{|c|c|c|c|c|}
\hline & $A(n=35)$ & $B(n=35)$ & $C(n=44)$ & Total $(n=114)$ \\
\hline Boys/girls & $16 / 19$ & $15 / 20$ & $18 / 26$ & $49 / 65$ \\
\hline Age (months) & $20.9(12.4)$ & $29.8(28)$ & $54.5(59.2)^{* * *}$ & $36.6(42.8)$ \\
\hline Range & $3.6-60.1$ & $2.7-127.1$ & $0.7-237.3$ & $0.7-237.3$ \\
\hline Weight (kg) & $11.4(4.2)$ & $13.5(8.9)$ & $17.6(13)^{\star *}$ & $14.5(10.0)$ \\
\hline Range & $6-30$ & $5.1-50$ & $3.2-50$ & $3.2-50$ \\
\hline Familial cases & $0(0 \%)$ & $4(11.4 \%)$ & $3(47 \%)$ & $7(6 \%)$ \\
\hline LVEF (\%) & $30(7)$ & $26(8) \dagger$ & $23(8)^{* * * *}$ & $26.4(8.5)$ \\
\hline Range & $15-43$ & $10-51$ & $14-48$ & $10-51$ \\
\hline LVEDV $\left(\mathrm{ml} / \mathrm{m}^{2}\right)$ & 91 (12) & $96(17)$ & $105(12)^{*}$ & $98.1(15.1)$ \\
\hline Range & $65-115$ & $45-130$ & $14-138$ & $10-138$ \\
\hline
\end{tabular}


an increase in LVEDV at follow up was considered to indicate persistent DCM of mild (LVEF $45-50 \%$ or LVEDV $70-80 \mathrm{ml} /$ $\mathrm{m}^{2}$ ), moderate (LVEF $35-45 \%$ or LVEDV $80-90 \mathrm{ml} / \mathrm{m}^{2}$ ), or severe $\left(\mathrm{LVEF}<35 \%\right.$ or LVEDV $\left.>90 \mathrm{ml} / \mathrm{m}^{2}\right)$ grade.

\section{Statistical analysis}

Numerical data are expressed as mean (SD). Discontinuous variables are reported as percentages. Differences between continuous or dichotomic variables were evaluated by means of factorial analysis of variance or contingency table, respectively. Freedom from adverse events was calculated with the Kaplan-Meyer product-limit method. Differences between curves were assessed by the Mantel-Cox test. Hazard ratios for adverse events were calculated by means of Cox's proportional hazard method. The unfavourable events considered in the survival and risk factors analyses were death and heart transplantation. A probability value of $\mathrm{p}<0.05$ was considered significant. The statistical package used was Statview 5.0 for Macintosh (SAS Institute Inc, Cary, North Carolina, USA).

\section{RESULTS}

\section{On admission}

The 114 children were grouped as follows: 35 children in group A, 35 in group B, and 44 in group C. There were more girls than boys in all three groups, and children in group $\mathrm{C}$ were older at diagnosis than were children in group A or B $(p=0.009)$. Children in group $C$ also had a worse left ventricular function than patients in group A or group B (table 1). Seven patients had a positive family cases (table 1) and four of these children died: two of four in group B and two of three in group C. The difference in mortality rate $(50 \%$ $v 33 \%$ ) was not significant ( $\mathrm{p}=0.22$ ), due probably to the small number of children with a positive family history.

\section{Survival}

The overall survival rate (groups A, B, and C) was $65 \%$. The cumulative (groups A and B) actuarial event-free survival probability was $0.96(0.04)$ at one year and $0.83(0.05)$ at 13 years. The event-free survival probability of children in group C was $0.61(0.07)$ at one year and $0.32(0.07)$ at 13 years. At 13 years, the event-free survival probability was 0.97 (0.03) for group A and 0.70 (0.08) for group B (fig 1). In group A, one of 35 patients $(3 \%)$ underwent heart transplantation and none died; in group B, 10 of 35 (29\%) either received a

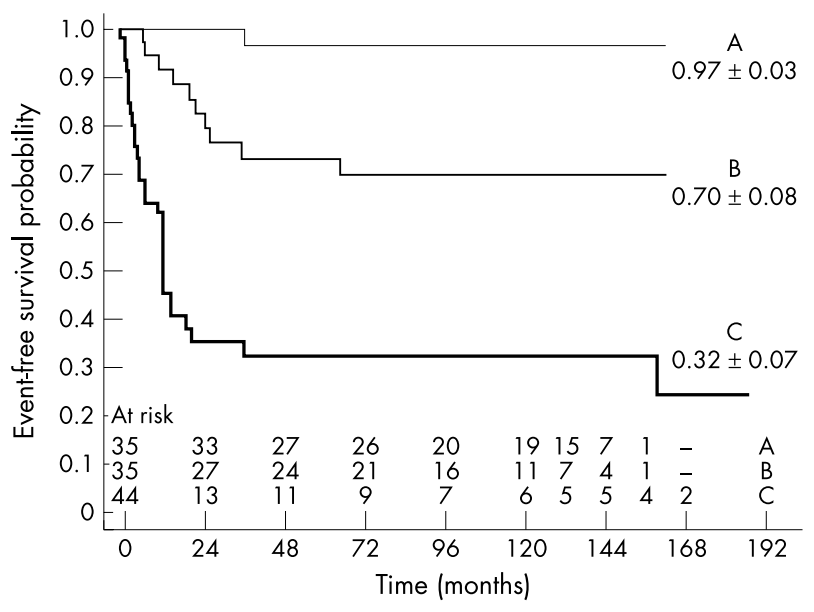

Figure 1 Plot of event-free survival probability for children with acute myocarditis (group A), borderline myocarditis (group B), and noninflammatory dilated cardiomyopathy (group $C$ ). For groups $B$ and $C$ the adverse events (transplant or death) are concentrated almost entirely in the first two years after the initial diagnosis. transplant (four) or died (six); and in group C, 30 of 44 (68\%) either received a transplant (27) or died (three), one of whom was the only neonate in our study. In groups B and C, the risk of death or of undergoing heart transplantation was concentrated almost entirely in the first two years after the initial diagnosis of DCM (fig 1). The analysis of proportional hazards showed that reduced LVEF, increased LVEDV, and the presence of non-inflammatory DCM were independent risk factors for earlier adverse events (table 2).

The mean value of wedge pressure was not significantly higher in children who died or received a transplant (10.4 (3.8) $\mathrm{mm} \mathrm{Hg}$ ) than in children who survived $(8.0$ (2.1) $\mathrm{mm} \mathrm{Hg})(\mathrm{p}=0.0982)$.

\section{Left ventricular function at follow up}

In groups $\mathrm{A}$ and $\mathrm{B}$, left ventricular function was either completely or partially recovered during the first six months after diagnosis. Table 3 shows mean LVEF and LVEDV. Patients in group $\mathrm{C}$ had less completely recovery of left ventricular function than children in groups $\mathrm{A}$ and $\mathrm{B}$. At the last follow up, some children had not completely recovered from DCM: seven of 34 (21\%) (five mild and two moderate) in group A; nine of 25 (36\%) (five mild, three moderate, and one severe) in group B; and nine of 14 (64\%) (three mild, one moderate, and five severe) in group $\mathrm{C}$ (table 3 ).

\section{Complications of EMB}

Of the 114 children who had an EMB, only one child had a pericardial effusion during the haemodynamic procedure due to a lesion caused by the bioptome. Prompt drainage resolved the effusion and no further complications were recorded during eight years of follow up.

\section{Evolution of histological pattern}

At the six month control EMB among survivors in group A, 19 of 26 patients $(73 \%)$ had complete or almost complete resolution of the myocarditis; the other seven $(27 \%)$ had persistent myocarditis. At 12 months, 14 of 18 children (78\%) had resolved or resolving myocarditis on EMB. The only child in this group who received a transplant had a stable pattern of persistent myocarditis, which eventually evolved into chronic DCM. In group B, 22 children among 25 survivors had the six month control EMB. Seventeen or the $22(77 \%)$ had a histological pattern of resolved to resolving myocarditis and five $(23 \%)$ had persistent inflammatory infiltrate. Thirteen had a third EMB at 12 months and 11 of them $(85 \%)$ had a pattern of resolution of the inflammatory process. The remaining two patients had evidence of chronic DCM but their cardiac function recovered satisfactorily.

\section{Recurrence of CHF}

CHF recurred in eight children (three in group A and five in group B) about three months after termination of the first six month cycle of immunosuppression, prompting resumption of immunosuppression. Of the three patients in group A, two had almost complete functional recovery (mild DCM) after the repeated treatment cycle but the third patient underwent heart transplantation. In group B, one patient had complete

\begin{tabular}{lcc} 
Table 2 & Independent predictors of time related outcome \\
\hline Covariate & Hazard ratio $(95 \% \mathrm{Cl})$ & $\mathrm{p}$ Value \\
\hline LVEF & $0.91(0.86$ to 0.97$)$ & 0.0045 \\
LVEDV & $1.03(1.00$ to 1.06$)$ & 0.0501 \\
DCM & $4.27(1.98$ to 9.20$)$ & 0.0002
\end{tabular}

$\mathrm{Cl}$, confidence interval; DCM, non-inflammatory dilated cardiomyopathy; LVEDV, left ventricular end diastolic volume indexed to body surface area; LVEF, left ventricular ejection fraction. 
Table 3 Recovery of left ventricular function in survivors

\begin{tabular}{|c|c|c|c|c|c|c|}
\hline \multirow[b]{2}{*}{ Group } & \multirow[b]{2}{*}{ Outcome } & \multirow[b]{2}{*}{ Number } & \multicolumn{2}{|l|}{ LVEF (\%) } & \multicolumn{2}{|c|}{ LVEDV $\left(\mathrm{ml} / \mathrm{m}^{2}\right)$} \\
\hline & & & Admission & Follow up & Admission & Follow up \\
\hline \multirow[t]{3}{*}{$\bar{A}$} & Normal LVF & 27 (77\%) & 31 (8) & $61(4)$ & $88(12)$ & $60(10)$ \\
\hline & Reduced LVF & $7(20 \%)$ & $30(6)$ & $53(8)$ & 99 (7) & $86(8)$ \\
\hline & Death/HTx & $1(3 \%)$ & 31 & NA & 90 & NA \\
\hline \multirow[t]{3}{*}{ B } & Normal LVF & $16(46 \%)$ & $26(7)$ & $60(4)$ & $99(13)$ & $61(9)$ \\
\hline & Reduced LVF & $9(26 \%)$ & $32(9)$ & 51 (8) & 84 (19) & 84 (15) \\
\hline & Death/HTx & $10(28 \%)$ & $22(7)$ & NA & $103(16)$ & NA \\
\hline \multirow[t]{3}{*}{ C } & Normal LVF & $5(12 \%)$ & 26 (12) & $57(3)$ & $92(9)$ & $65(13)$ \\
\hline & Reduced LVF & $9(21 \%)$ & $32(9)$ & $42(10)$ & $99(20)$ & $93(9)$ \\
\hline & Death/HTx & $30(68 \%)$ & 20 (4) & NA & $109(7)$ & NA \\
\hline
\end{tabular}

HTx, heart transplantation; LVF, left ventricular function; LVEDV, left ventricular end diastolic volume indexed to body surface area; LVEF, left ventricular ejection fraction; NA, not applicable.

recovery of left ventricular function and two had persistent mild DCM (to date these patients have not been completely weaned from immunosuppression because of repeated recurrences of CHF after suspension of prednisone).

\section{Side effects}

No patient had any bacterial infection and the creatinine concentrations were consistently in the normal range. Apparently no new viral infection occurred during the treatment cycle. No children had arterial hypertension or hyperglycaemia. Hirsutism was recorded commonly but it invariably receded after the termination of immunosuppression.

\section{DISCUSSION}

The series of paediatric cases of DCM presented here is the largest reported to date (114 patients) and has the longest follow up (up to a median of 5.7 years). Our overall survival rate was $65 \%$. The mean combined survival rate of three different series of children ${ }^{4-6}$ treated only conventionally was $44 \%$ at meta-analysis. These rates differ significantly in a contingency table comparison $(\mathrm{p}=0.0045)$.

Clinical data at diagnosis showed that the youngest children were more likely to have acute florid myocarditis and also presented with the best heart function. These children had the best outcome, in terms of both event-free survival rate (mean (SD) $0.97(0.03)$ ) and functional recovery of the heart (normal or close to normal in $90 \%$ of the cases). On the other hand, children in group $\mathrm{C}$ were the oldest, were more likely to have no apparent signs of cardiac inflammation, and had the worst cardiac function.

In a large proportion of children with either acute or borderline myocarditis the inflammatory process resolved, as shown at follow up by EMB, coupled with good left ventricular function. However, there were also a few patients with normal, or almost normal, left ventricular function but with persistent inflammation at EMB. In the latter group, the results of EMB alone could have led to an erroneous overestimation of the actual extension of the myocarditic process.

Compared with the group of patients with acute florid myocarditis, children with borderline myocarditis had a lower rate of event-free survival $(0.70(0.08))$ and a lower incidence of functional recovery of the heart $(60 \%)$ to normal or near normal levels. It is unclear why patients with more intense inflammation and with the same immunosuppressive regimen are more likely to improve. Borderline myocarditis, with scarce inflammatory cells and no myocyte necrosis, may be the burn-out stage of an acute florid myocarditis that has already evolved into chronic myocarditis. ${ }^{22}$ If this assumption is correct, then the immunosuppressive agent would be given too late because the disease would already have entered the stage of progressive fibrosis and unrecoverable DCM, a hypothesis that has already been proposed by others. ${ }^{23}$

Children with the non-inflammatory form of DCM receiving only conventional treatment had worse clinical results than the two groups with myocarditis in terms of both eventfree survival $(0.32 \vee 0.83)$ and cardiac functional recovery (36\% $v 72 \%)$. Although we may have missed foci of myocarditis with our tissue sampling procedure (so called false negative biopsies ${ }^{24}$ ), our definition of non-inflammatory DCM was reached after scanning several sections of four to five biopsies. It has been suggested that the non-inflammatory form of DCM is the end stage result of a process that starts as florid myocarditis, moves towards borderline myocarditis, and ends up in fibrosis. However, certain questions remain. A three stage progressive pathological process seems unlikely because patients with non-inflammatory DCM seem to have no acute symptoms at the time when the postulated acute florid myocarditis should first appear. In view of this, we suggest that there are two distinct types of DCM: one that is initially characterised by various degrees of inflammation leading to fibrosis; and one that has the fibrotic process as its primary pathological feature.

It appears that the first two years after diagnosis are crucial for children with DCM, in that favourable events (almost full recovery of cardiac functions) and the worst outcomes (heart transplantation or death) both occur during this relatively short period of time, regardless of whether the children are affected by inflammatory or by non-inflammatory DCM. After this period, only a few children (eight of 70 in our series) with acute or borderline myocarditis had recurrences. Of these eight patients with recurrent CHF, six recovered immediately after reinstitution of the immunosuppression.

The data presented so far have indicated that immunosuppression and immunomodulation (IgG administration) given during the first year after diagnosis ${ }^{18}$ are associated with a high rate of survival of children with myocarditis, though the survival rate is higher with immunosuppression than with immunomodulation $(0.96 v 0.84)$. When adult patients with myocarditis took part in a randomised study with immunosuppressive compared with conventional treatment, $44 \%$ survived in both groups in a five year follow up. ${ }^{13}$ These results differ from the results of our study, in which $83 \%$ of similarly treated children with myocarditis survived ( 13 year follow up). Several reasons may account for these differences. In the adult study, ${ }^{13}$ an unspecified number of patients did not start immunosuppressive treatment at diagnosis, while in all of our paediatric cases treatment was begun shortly after diagnosis. Moreover, in the same study ${ }^{13}$ myocarditis was misdiagnosed in $39 \%$ of cases (most probably idiopathic DCM), which may also account for the decreased survival in the adult group. ${ }^{25}$ If all the adults with bona fide myocarditis had received immunosuppressive treatment early after diagnosis, it is possible that the survival 
rate would have been higher, similar to that observed among children. On the other hand, it is also possible that children have a better potential for recovery.

In an observational paediatric study, ${ }^{18}$ children with myocarditis (biopsy proven) and receiving conventional treatment exclusively had a survival probability at one year of 0.60. In similar observational studies with adult patients with DCM and similarly treated with conventional treatment, ${ }^{7}$ the five year survival was 0.56 in the group as a whole-that is, patients with histologically proven myocarditis or non-inflammatory DCM. What does emerge quite clearly from all the observational and immunosuppression studies carried out so far is that $60 \%$ of children and $50 \%$ of adult patients with myocarditis tend to recover spontaneously. Therefore, about $50 \%$ of our paediatric patients may have been treated unnecessarily with immunosuppression. However, on a positive note, one can also say that at least 25$30 \%$ of our treated children would have died or needed heart transplantation, had they not received immunosuppression. Histological or serological markers may possibly reliably predict which children would benefit from immunosuppression and which would otherwise recover spontaneously. Some of the markers already identified, such as cardiac autoantibodies, ${ }^{26-28}$ viral products both in peripheral blood and in the $\mathrm{EMB}^{28}$ or inappropriate expression of HLA molecules on the affected cardiac tissue ${ }^{1920}$ would help, separately or together, to this end. In fact, with such markers, it would be possible to stratify patients for immunosuppressive or conventional treatment. Whether the high rate of treatment success in our children with myocarditis is correlated with the absence of myocardiotropic viruses and the presence of cardiac autoantibodies, as recently observed in an adult population, ${ }^{28}$ has not been substantiated.

Although we are the first to be aware that a limitation of our study is the lack of a matched control group, our earlier results, ${ }^{15}$ as well as those of others, ${ }^{16}{ }^{17}$ obtained after the introduction of immunosuppression were so encouraging that it seemed almost unethical at that time to propose a randomised trial. Nowadays, we still believe that a simple randomisation would be of some concern, in the view of such high survival rates, unless a more accurate characterisation of myocarditis would differentiate the potential responders to immunosuppression from the non-responder population. ${ }^{19} 28$ For this purpose, we started to analyse the myocardial biopsies retrospectively for DNA virus genome and we now systematically analyse frozen biopsies for RNA virus genome. Similarly, sera are now tested for the presence of cardiac autoantibodies.

\section{ACKNOWLEDGEMENTS}

This work was partially supported by a grant of the Italian Ministry of Health. We thank Mrs Andreina Santoro for patiently editing the manuscript. We also thank Ms Jean Ringrose for her consultation on the English language.

\section{Authors' affiliations \\ M Giulia Gagliardi, M Bevilacqua, B Leonardi, Departments of} Cardiology and Cardiac Surgery, Ospedale Pediatrico Bambino Gesù, Scientific Institute, IRCCS, Rome, Italy

R Boldrini, F Diomedi Camassei, Department of Pathology, Ospedale Pediatrico Bambino Gesù

A G Ugazio, Department of Paediatrics, Ospedale Pediatrico Bambino Gesù

A Fierabracci, Laboratory of Autoimmunity, Ospedale Pediatrico Bambino Gesù

G F Bottazzo, Scientific Directorate, Ospedale Pediatrico Bambino Gesù
C Bassano, Department of Cardiac Surgery, Tor Vergata University, Rome, Italy

Presented in part at the Scientific Sessions of the American Heart Association, Chicago, 17-20 November 2002 and published as an abstract in Circulation 2002;106;:11719.

\section{REFERENCES}

1 Kawai C. From myocarditis to cardiomyopathy: mechanisms of inflammation and cell death: learning from the past for the future. Circulation 1999:99:1091-100.

2 Feldman AM, McNamara D. Myocarditis. N Engl J Med 2000;343: 1388-98.

3 Aretz HT, Billingham ME, Edwards WD, et al. Myocarditis: a histopathologic definition and classification. Am J Cardiovasc Pathol 1987;1:3-14.

4 Taliercio CP, Seward JB, Driscoll DJ, et al. Idiopathic dilated cardiomyopathy in the young: clinical profile and natural history. J Am Coll Cardiol 1985;6:1126-31.

5 Griffin ML, Hernandez A, Martin TC, et al. Dilated cardiomyopathy in infants and children. J Am Coll Cardiol 1988;11:139-44.

6 Chen SC, Nouri S, Balfour I, et al. Clinical profile of congestive cardiomyopathy in children. J Am Coll Cardiol 1990;15:189-93.

7 Grogan M, Redfield MM, Bailey KR, et al. Long-term outcome of patient with biopsy-proved myocarditis: comparison with idiopathic dilated cardiomyopathy. J Am Coll Cardiol 1995:26:80-4.

8 Kishimoto C, Abelmann WH. In vivo significance of T cells in the development of coxsackie virus B3 myocarditis in mice: immature but antigen-specific T cells aggravate myocardial injury. Circ Res 1990;76:589-98.

9 Martino TA, Liu P, Sole MJ. Viral infection and the pathogenesis of dilated cardiomyopathy. Circ Res 1994;74:182-8.

10 Knowlton KU, Badorff C. The immune system in viral myocarditis: maintaining the balance. Circ Res 1999;85:559-61.

11 Opavsky MA, Penninger J, Aitken K, et al. Susceptibility to myocarditis is dependent on the response of T lymphocytes to coxsackieviral B3. Circ Res 1999:85:551-8.

12 Parrillo JE, Cunnion RE, Epstein SE, et al. A prospective, randomized, controlled trial of prednisone for dilated cardiomyopathy. N Engl J Med 1989;321:1061-8.

13 Mason JW, O'Connell JB, Herskowitz A, et al. A clinical trial of immunosuppressive therapy for myocarditis. N Engl J Med 1995;333:269-75

14 Camargo PR, Snitcowsky R, da Luz PL, et al. Favorable effects of immunosuppressive therapy in children with dilated cardiomyopathy and active myocarditis. Pediatr Cardiol 1995;16:61-8.

15 Gagliardi MG, Bevilacqua M, Squitieri C, et al. Dilated cardiomyopathy caused by acute myocarditis in pediatric patients: evolution of myocardia damage in a group of potential heart transplant candidates. $J$ Heart Lung Transplant 1993;12(suppl):S224-9.

16 Kleinert S, Weintraub RG, Wilkinson JL, et al. Myocarditis in children with dilated cardiomyopathy: incidence and outcome after dual therapy immunosuppression. J Heart Lung Transplant 1997; 16:1248-54.

17 Lee KJ, McCrindle BW, Bohn DJ, et al. Clinical outcome of acute myocarditis in childhood. Heart 1999:82:226-33.

18 Drucker NA, Colan SD, Lewis AB, et al. Globulin treatment of acute myocarditis in the pediatric population. Circulation 1994;89:252-7.

19 Wojnicz R, Nowalany-Kozielska E, Wojciechowska C, et al. Randomized, placebo-controlled study for immunosuppressive treatment of inflammatory dilated cardiomyopathy: two-year follow-up result. Circulation $2001 ; 104: 39-45$

20 Caforio AL, Stewart JT, Bonifacio E, ef al. Inappropriate major histocompatibility complex expression on cardiac tissue in dilated cardiomyopathy: relevance for autoimmunity? J Autoimmun 1990:3:187-200

21 Rein AJ, Colan SD, Parness IA, et al. Regional and global left ventricular function in infants with anomalous origin of the left coronary artery from the pulmonary trunk: preoperative and postoperative assessment. Circulation 1987; 75:1 15-23.

22 Angelini A, Crosato M, Boffa GM, et al. Active versus borderline myocarditis: clinicopathological correlates and prognostic implications. Heart 2002;87:210-5.

23 Liu PP, Mason JW. Advances in the understanding of myocarditis. Circulation 2001;104:1076-82.

24 Chow LH, Radio SJ, Sears TD, et al. Insensitivity of right ventricular endomyocardial biopsy in the diagnosis of myocarditis. J Am Coll Cardiol 1989; 14:915-20.

25 Parrillo JE. Myocarditis: how should we treat in 1998? J Heart Lung Transplant 1998;17:941-4.

26 Caforio AL, Bonifacio E, Stewart JT, et al. Novel organ-specific circulating autoantibodies in dilated cardiomyopathy. J Am Coll Cardiol 1990; 15: 1527-34.

27 Staudt A, Bohm M, Knebel F, et al. Potential role of autoantibodies belonging to the immunoglobulin G-3 subclass in cardiac dysfunction among patients with dilated cardiomyopathy. Circulation 2002;106:2448-53.

28 Frustaci A, Chimenti C, Calabrese F, et al. A Immunosuppressive therapy for active lymphocytic myocarditis: virological and immunologic profile of responders versus nonresponders. Circulation 2003;107:857-63. 